\title{
Research on the topology control algorithm to maximize the Ad Hoc life cycle
}

\author{
Wenbo Zhang*, Yongxin Feng, Xiaobo Tan and Lidong Fu \\ School of Information Science and Engineering, Shenyang Ligong University, Shenyang 110159, China.
}

Received 3 January, 2013; Accepted 24 Mar, 2014

\begin{abstract}
For Ad Hoc network, nodes move randomly with limited energy and self-organization; there is multi-hop of complex network, however, in order to extend the life cycle of Ad Hoc network effectively. This research was aimed at improving LEACH algorithm clustering on Ad Hoc network, constructing Ad Hoc network topology based on small world, making nodes that can still continue to maintain communication under the circumstances of communication link failure, appearance, disappearance and move. Namely, the work aims to improve the stability of the network topology and overall network antidestroying. The simulation experiment verifies the correctness and effectiveness of algorithm.
\end{abstract}

Key words: Ad Hoc network, complex network, small world network, life cycle, survivability.

\section{INTRODUCTION}

In this paper, an approach for topology control to extend the lifetime of Ad Hoc network was studied, in order to synthesize various factors as far as possible to maximize network survival time, and to make node maintain communications under the circumstances such as appearance, disappearance, movement and communication link failure. There are some ways about survivability study as follows. Firstly, the network topology structure is designed in the beginning, and during the design, various factors that prolong the survival time of the network, maintain network stability and improve the overall performance of the network are all considered. Secondly, Ad Hoc network is self-organizing and multihop. It has some characteristics of complex network. So the complex network theory could be used in Ad Hoc network to enhance its survivability. Finally, in order to maintain the stability of the network, the node or link failure and other factors such as redundancy, reconstruction and other measures should be considered (Liu et al., 2011, 2012; Mistra and Thomasinous, 2010; Ok et al., 2009; Ren et al., 2011; Uster and Lin, 2011).

The Ad hoc network topology is flat. So in order to enhance its survivability, it should have high connectivity between each node, that is to say, there are many paths between two nodes, if one path fails, start the other one. To make node strong connected, it is essential to increase the transmission power of the node, enlarge transmission range of nodes and cover more nodes. But increasing the transmission power of the node consumes its energy and shorts the network lifetime, so the energy consumption and network survivability is contradictory. This paper constructs a hierarchical network, which has different clustering algorithms by means of cluster head election, and synthesizes the residual energy of node, mobility, deviation of node degree (cluster size) and other factors to cluster (Wendi et al., 2002; Xiaohua et al., 2005; 


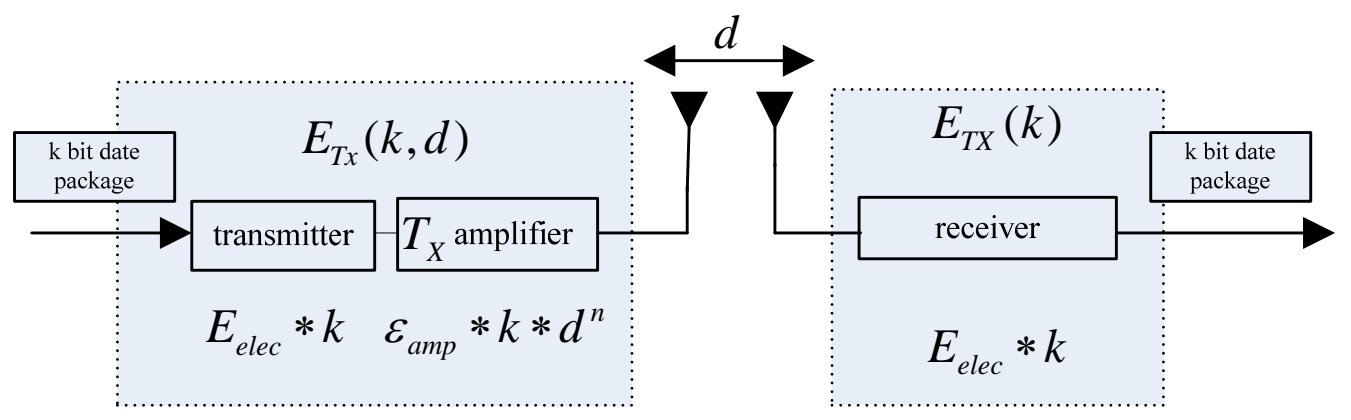

Figure 1. Radio energy model.

Xu and Liang, 2011).

\section{LOW-ENERGY ADAPTIVE CLUSTERING HIERARCHY (LEACH), MODIFIED WEIGHTED CLUSTERING ALGORITHM (MWCA) CLUSTERING ALGORITHM ANALYSIS}

\section{The process of the cluster head election}

At this stage, cluster head election algorithm combines low energy adaptive clustering hierarchical (LEACH) improvement algorithm in wireless sensor networks with an on-demand weighted clustering algorithm (WCA). That is, the clustering algorithm $\left\{\begin{array}{l}\text { LEACH }(T=0) \\ W C A(T>0)\end{array}\right.$

The threshold for LEACH algorithm:

$$
T_{n}=\frac{P}{1-P[r \bmod (1 / P)]} \quad n \in G
$$

The calculation method of on-demand weighted clustering algorithm:

weight $=a E_{V_{\text {resident }}}+b / M_{V}+c / D_{V}$

$E_{V_{\text {resident }}}$ is the remaining energy of nodes, which was obtained from node energy model (Yao-Chung et al., 2006) .

Figure 1 shows the diagram of energy model of nodes. Assuming a simple model for the energy consumption of wireless hardware, in this model, the sending end energy consumption includes sending radio electronics and amplifier energy consumption, the receiving end energy consumption includes receiving radio electronic energy consumption as shown in Figure 1. For this description, there are two kinds of channel model, free space model (energy loss and transmission distance is proportional to the square) and multi-path fading model (the energy loss is proportional to transmission distance to the fourth power), conversion between the two models is based on the distance between the sending and the receiving end, and power control can be set by the appropriate power amplifier and change the distance between the sending and receiving end. If the distance is less than the threshold value $d_{0}$, we use the free space model, otherwise, we use multi-path attenuation model. Therefore, when the distance between sending and receiving end is $d$ with a $l$ - bit message, the radio energy consumption is:

$$
\begin{aligned}
& E_{T X}(1, d)=E_{T X-\text { elec }(1)}+E_{T_{X}-\text { amp }}(1, d) \\
& =\left\{\begin{array}{l}
1 E_{\text {elec }}+1 \varepsilon_{f_{S}} d^{2}, d<d_{0} \\
1 E_{\text {elec }}+1 \varepsilon_{\text {amp }} d^{4}, d \geq d_{0}
\end{array}\right.
\end{aligned}
$$

To receive this message, the radio energy consumption:

$$
E_{R x}(1)=E_{R x-e l e c}(1)=1 E_{\text {elec }}
$$

The energy consumption of sending and receiving end is the sum of the total energy consumption of the node, the difference of the initial energy and total energy consumption is the residual energy of the node.

The $M_{V}$ presents the local mobility of the node, it can be calculated by a set of relative mobility metric values from all the neighboring nodes.

For the relative mobility metric value of node $u$ to node $\checkmark$ :

$$
M_{V}^{\text {rel }}(u)=10 \lg \frac{R_{X} P_{r_{u \rightarrow V}^{\text {new }}}}{R P_{X} P_{r_{u \rightarrow V}^{\text {old }}}} \text {, if } R_{X} P_{r_{u \rightarrow V}^{\text {new }}}<R P_{X} P_{r_{u \rightarrow V}^{\text {old }}}
$$

then $M_{V}^{r e l}(u)<0$, it means that the two node are far away from each other, contrary, if $R_{X} P_{r_{u \rightarrow V}^{\text {ner }}}>R_{X} P_{r_{u \rightarrow V}^{o l d}}$, it shows that the two nodes are close to each other. If a node $v$ has $m$ neighbor nodes, there are $m$ value $M_{V}^{r e l}$, the local mobility value is the difference between the relative mobility value of all neighbors and 0 , that is: 


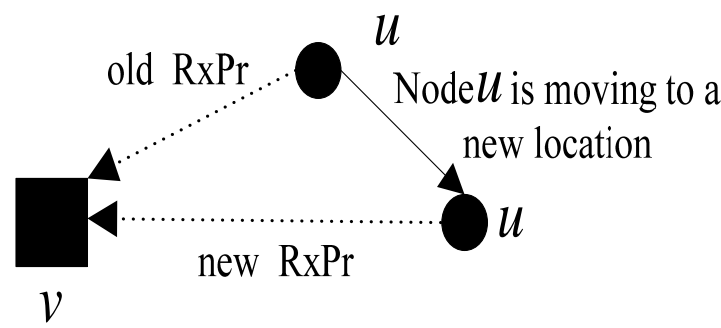

Figure 2. Local movement and relative movement.
$M_{V}=\operatorname{var}_{0}\left\{M_{V}^{r e l}\left(u_{i}\right)\right\}_{i=1}^{m}=E\left[\left(M_{V}^{r e l}\right)^{2}\right], \quad M_{V}$ is calculated. If $M_{V}<0$, it indicates that the node is away from neighbors, if $M_{V}>0$, it shows the node is close to neighbor nodes (Figure 2).

$D_{V}$ means the difference between node degree (number of neighbor nodes) and the ideal degree, as the $D_{V}=\left|d_{V}-M\right|$, where $d_{V}$ denotes the number of neighbor nodes. The calculation formula of $d_{V}$ is as follow:

$$
d_{V}=|N(v)|=\sum_{V^{\prime}, v \in V, V^{\prime} \neq V}\left\{\operatorname{dist}\left(V, V^{\prime}\right)\right\}<t_{\text {range }}
$$

here $t x_{\text {range }}$ represents the transmission range of the nodes.

A weight implies the combination weight of node, namely to the ability to act as cluster head node. For the weight factor $a, b$ and $c$, we set $a>b>c$ in this thesis, and they satisfy $a+b+c=1$. Based on the calculation formula of combination weight, we are mainly considering three factors: the energy of node, the mobility of node (cluster stability) and the size of cluster (node degree). Among them, if the local mobility and the difference between the node degree and the ideal degree are small, the possibility of a node becoming cluster head node is big, and the stability of clusters is strong. If the remaining energy is large, the probability of the node becoming cluster head is high. Therefore, by calculating, it shows that we should choose the larger weight as the cluster head node.

\section{The initial stage of establishing a cluster}

During the initial process of creating a cluster in mobile Ad Hoc networks, a mobile node first starts and then enters into networking; and at last forms a relatively stable cluster structure within the scope of the entire network. When we initially create a cluster or a new node join in, through the initialization process, deciding what role of the node should play in.

When a system boots, a globally unique node ID is given, all nodes are randomly well-distributed in a region with the same initial energy, the energy consumption of mobile nodes are not the same each round. In the first round, we use the LEACH algorithm, namely $t=0$, during a short time of executing the clustering algorithm, the mobile node in Ad Hoc network nodes do not move. Each node is randomly assigned a random number, comparing the random number with threshold, when the random number is less than the $T_{n}$, the node is selected as the cluster head node. Then, the node which was selected as the cluster head broadcast Hello message packet, which includes the serial number of the cluster head node. The non-cluster head nodes which received the broadcast message packet, choose to add to the strong signal clusters, according to the distance between them and the cluster head nodes, notify the cluster head, when cluster head node receives all the information added to the cluster, it will create a time division multiple access (TDMA) timing information and inform all the nodes within the cluster. In order to avoid the interference of signal from nearby cluster, the cluster head node can decide code division multiple access (CDMA) code of all nodes in the cluster, CDMA code along with the TDMA are sent at scheduled times, when a node within the cluster receives the message, it begins to enter the data communication phase. In this phase, each node has the trend to move in the cluster. We should consider this dynamic problem time discretization.

Assuming that each node has different energy consumptions in each round, namely the node's residual energy is different. In order to balance the energy consumption of all nodes, we should select each node to become a cluster head periodically so that each node has to the opportunity to act as the cluster head. In the second round of the cycle, we use the on-demand weighted clustering algorithm to initialize the node information along with the weight of the node. The weight of the node includes the current residual energy, local mobility and the degrees (one-hop neighbor node of the 
nodes), the number of cluster head and the ID information of the cluster heads. In addition, we also initialize neighbor list information of the node and the cluster head table information.

The node should put its own ID, transmit and receive signal power into broadcast message, and broadcast to one-hop neighbors. When neighbor nodes receive broadcast message of that node, it checks its own neighbor list. If the node has already existed in the neighbor list, update is made to the information in neighbor list of the node with mobile information and update time. Node should be added, if it does not exist in the neighbor list. After a node transmits broadcast message a period of time, when assuming that it has received the message from all neighbors, that is to say, there is no new neighbor node to be added to the neighbor list of the node in a period of time, it begins to calculate its own weights.

First through the energy model of the node, when we calculate weights, the residual energy $E_{\text {resident }}$ is obtained. The node goes through its own neighbor list. Through the neighbor list stored in, the node receives the same at different times of signal power; we can figure out $M_{V}^{r e l}(u)$, along with $M_{V}$.

The total number of neighbors is extracted from the neighbor list, $d_{V}$ is calculated and then we obtain the $D_{V}=\left|d_{V}-M\right|$. According to the calculated $E_{\text {resident }}, M_{V}$, $D_{V}$ and the importance of the three factors that are previously set as $a, b 、 c$ to calculate the combination weight of each node, each node puts combination weight and node ID in the node weight broadcast message, and broadcasts to one-hop neighbors.

After each node receives the neighbor node weight message, they update their neighbor list information. Within a period of time, if the node does not receive the node weight message in the original neighbor list, the node should be deleted from original neighbor list; if a node receives weights message in original neighbor list, it is necessary to update the neighbor node weight; if a node receives weight message instead of the original neighbor list, we have to discard it.

After the node sends weight message a period of time, if all weights in the neighbor node list are updated, the node determines whether its weight are larger than all the neighbors' nodes or not; if the weights are equal, the node compares whether node ID is smaller than the neighbor's. If it satisfies this condition, node sends a message that it acts as the cluster head, otherwise waits for news of neighbor nodes becoming cluster heads.

For ordinary nodes which have not be elected as cluster head, if they receive the message of neighbors becoming cluster head, its state turn into cluster members, next to update their cluster head table and retreat from cluster head election. Otherwise, the node sets itself to the cluster head, and sends a message that it becomes the cluster head. If there are still nodes in the initial state, following the round cycle to execute cluster head election process until the status of all nodes turn into cluster head or cluster members is necessary. This facilitates selecting cluster head periodically so that each node has opportunity to become a cluster head, and thus the energy consumptions of nodes are more balanced.

\section{INTER-CLUSTER COMMUNICATION}

The inter-cluster communications connect cluster heads to form a ring network, for each node can become a cluster head, the nodes in ring network also periodically change. We construct the small world network based on this topology according to two properties of the small world network: the big clustering coefficient and the small shortest path. Firstly, long links were constructed; where $\mathrm{N}$ represents the number of vertex ring, the $\mathrm{P}$ represents the random connection probability, and the $\mathrm{K}$ is the number of adjacent nodes, according to the formula, the average path length: $L=\frac{N}{K} f(N K P)$, and the clustering coefficient $\quad C=\frac{3(k-1)}{2(2 k-1)}(1-p)^{3} \quad, \quad$ according to the parameters of $\mathrm{N}, \mathrm{P}, \mathrm{K}$ (both determine the number of long links). The purpose is to construct the topology with the shortest path, while the clustering coefficient is used to describe the node graph or network integration group coefficient. The network is divided into clusters on the whole, and its clustering coefficient is very high. Thus on the whole, we construct the topology of Ad Hoc network based on small world network, each cluster can communicates with each other. The topology diagram of Ad Hoc networks is based on small world network (Figure 3).

After the cluster is created, it is essential for the reasonable maintenance mechanism of clusters to try to maintain the stability of the cluster structure, due to the limited energy and the natural failure or link failure and the mobility of the node. At the moment of the node failure or link failure, node can still maintain the ability of communication, namely to prolong the network life cycle. Therefore, in this paper, the maintenance of clusters are mainly a node joins, disappears and moves. Node joining makes use of network reconfiguration, in order to quickly start backup node to backup link if node disappears and the link fails.

\section{LOW-ENERGY ADAPTIVE CLUSTERING HIERARCHY (LEACH) AND MODIFIED WEIGHTED CLUSTERING ALGORITHM (MWCA) SIMULATIION AND ANALYSIS}

The simulation model comprised 50 nodes, randomly distributed in the range of $100 \mathrm{~m} * 100 \mathrm{~m}$; the network is divided into 10 clusters, namely 10 cluster heads. The 


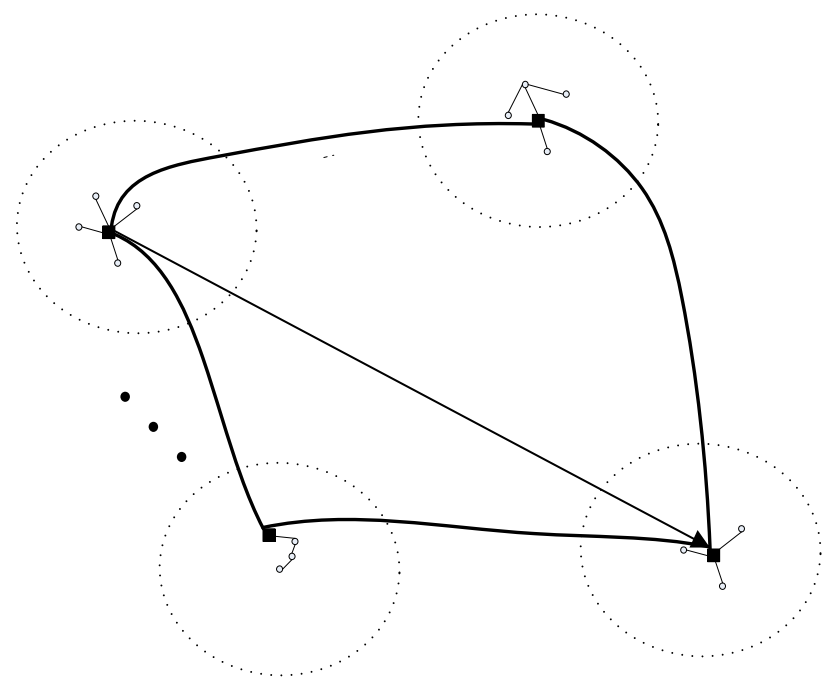

簇四簇首成员—长链接

Figure 3. Topology diagram of Ad Hoc networks based on small world network.

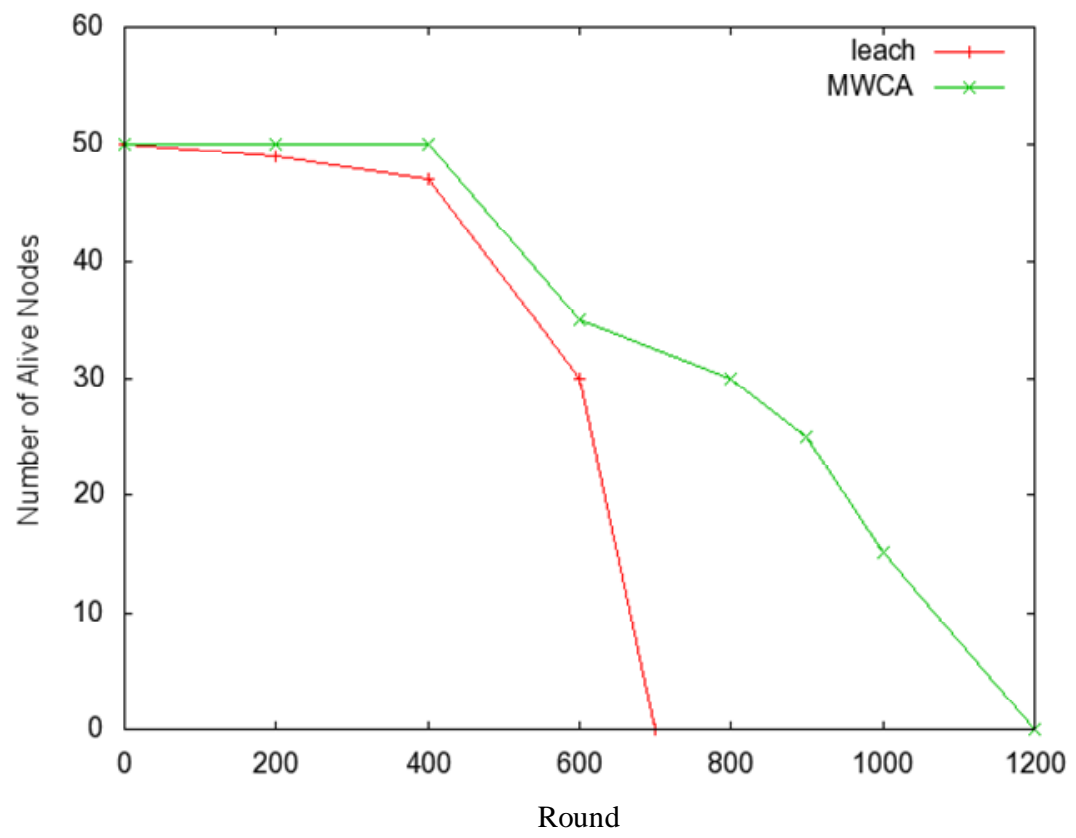

Figure 4. The diagram of election times and survival node numbers.

initial energy of the nodes is $1 \mathrm{~J}$, the actual connection degree is $M=4$, the total simulation time is $800 \mathrm{~min}$. The related parameters of the small world network is set to $\mathrm{N}$ $=10, \mathrm{P}=0.2, \mathrm{~K}=4$ (adjacent nodes), each round of cycle interval is $50 \mathrm{~s}$, a total of 960 rounds.

Figure 4 shows the relationship between curve of cluster heads election rounds and the remaining nodes in Ad Hoc network based on the small world. From Figure 4 using the simulation of LEACH algorithm, all nodes are dead after 700 rounds of the cluster head election, but applying the LEACH and MWCA clustering algorithm we put forward, the life cycle of the node is 1200 rounds, not 


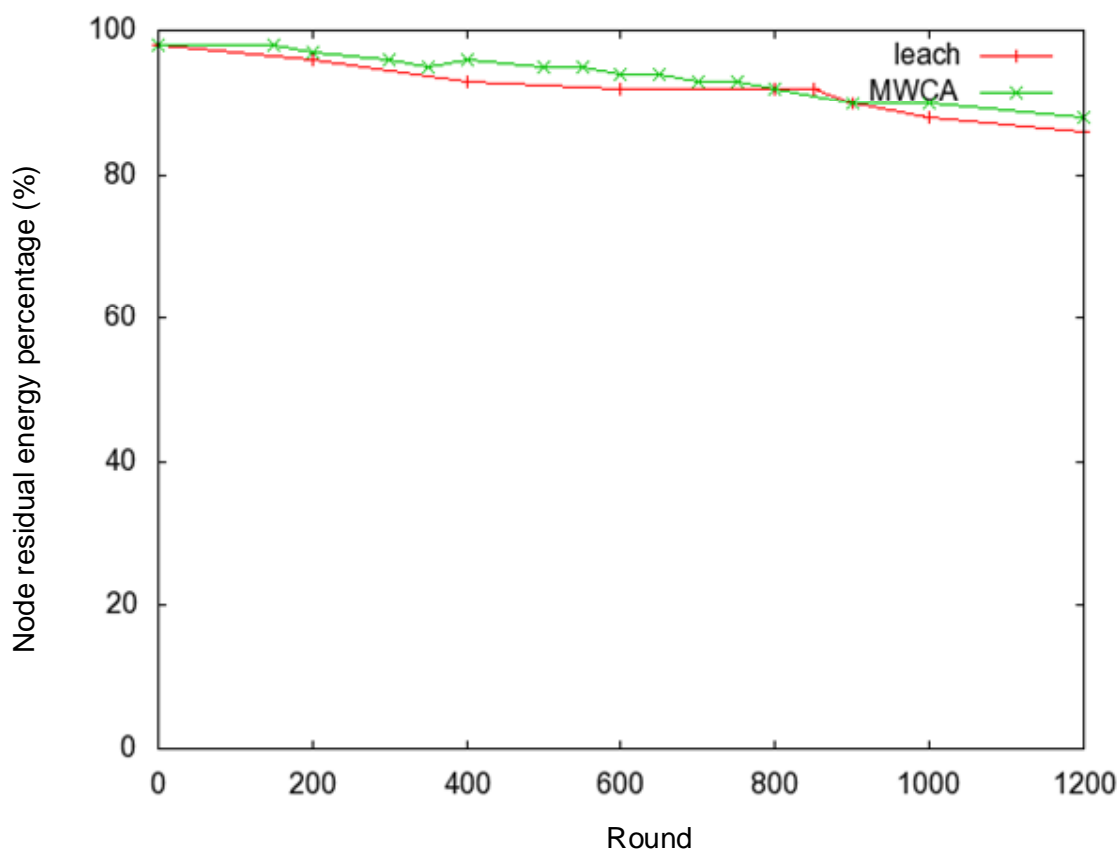

Figure 5. The diagram of election times and network node residual energy percentage.

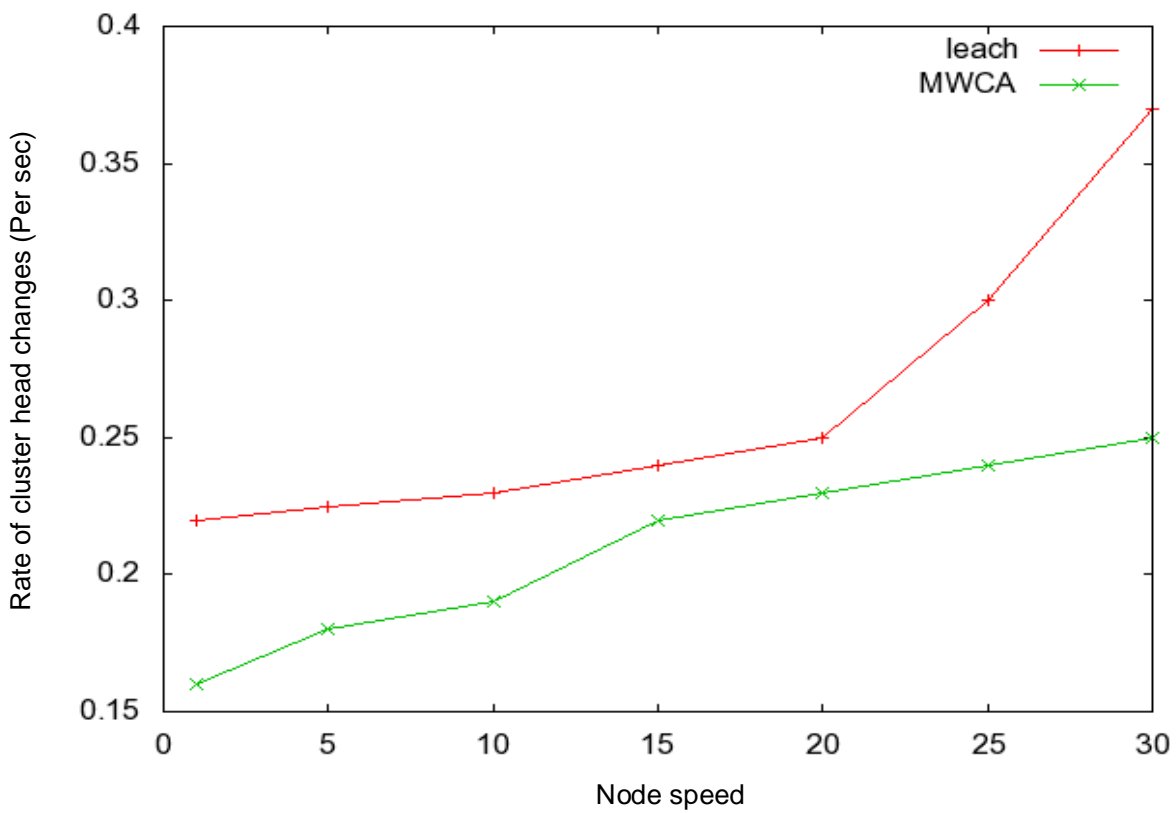

Figure 6. The diagram of node speed and rate of cluster head changes.

because of the hasten death of nodes due to the excessive energy consumption of the cluster head node, to improve the stability of the network topology and the survivability of overall network, but because of the cluster heads election algorithm proposed in this paper considers the residual energy and mobility of the node and the maximum number of nodes for cluster head nodes handling, to make the energy consumption of nodes in the network more homogeneous and stable.

From Figures 5 and 6 shown, the LEACH algorithm does not consider the residual energy and mobility of nodes, although each node has the probability of acting as cluster head, individual cluster head nodes accelerate the death due to excessive energy consumption. As the 
node speed increases, the cluster head change rate is larger; if the entire cluster is not stable, maintaining the stability of the cluster and network topology will consume more energy, while the algorithm proposed in this paper considers the residual energy and mobility of nodes, the nodes which have much residual energy and low moving rate act as cluster head, the cluster head is more stable, the lifetime of all nodes in the whole network is longer, according to the passage above, we know that the LEACH and MWCA clustering algorithm in the Ad Hoc network based on the small world is more stable, the antidestroying ability of the whole Ad Hoc network is stronger.

\section{CONCLUSIONS}

In this research, the approach to hierarchical topology control in maximizing network lifetime of Ad Hoc network based on small world was put forward. In the process of the cluster head election, we do not only comprehensively consider the residual energy of the node, mobility of the node and the maximum number for cluster head node to handle, but also make use of smallworld model to maintain the stability of the node and to enhance the anti-destroying ability of the network. The simulation results show that compared with other clustering algorithms, the proposed clustering algorithm in this paper can prolong the network lifetime effectively.

\section{Conflict of Interests}

The author(s) have not declared any conflict of interests.

\section{ACKNOWLEDGEMENTS}

This paper is sponsored by New Century Program for Excellent Talents of Ministry of Education of China and Shenyang Ligong University Computer Science and Technology Key Discipline Open Foundation.

\section{REFERENCES}

Liu A, Ren J, Li X, Chen Z, Shen X (2012). Design principles and improvement of cost function based energy ware routing algorithms for wireless sensor networks. Comput. Netw. 56:1951-1967. http://dx.doi.org/10.1016/j.comnet.2012.01.023

Luo D, Zhu X, Wu X, Chen G (2011). Maximizing lifetime for the shortest path aggregation tree in wireless sensor networks. In: Proc. of IEEE INFOCOM '1 1, 2011, April 10-15, pp. 1566-1574. PMid:21714026 PMCid:PMC3190151

Mistra S, Thomasinous PD (2010). A simple, least-time and energy efficient routing protocol with one level data aggregation for wireless sensor networks. Syst. Softw. 83:852-860. http://dx.doi.org/10.1016/j.jss.2009.12.021

Ok CS, Lee S, Mitra P, Kumara S (2009). Distributed energy balanced routing for wireless sensor networks." Comput. Ind. Eng. 57:125-135. http://dx.doi.org/10.1016/j.cie.2009.01.013

Ren F, Zhang J, He T, Lin C, Das SK (2011). Energy balanced routing protocol for data gathering in wireless sensor networks. IEEE Trans. Parallel Distrib. Syst. 22(12). http://dx.doi.org/10.1109/TPDS.2011.40

Uster H, Lin H (2011). Integrated topology control and routing in wireless sensor networks for prolonged network lifetime. Adhoc Netw. 9(5):835-851. http://dx.doi.org/10.1016/j.adhoc.2010.09.010

Wendi BH, Anantha PC, Hari B (2002). An Application-Specific Protocol Architecture for Wireless Microsensor Networks. IEEE Trans. Wireless Commun. 1(4):660-670. http://dx.doi.org/10.1109/TWC.2002.804190

Xiaohua J, Dongsoo K, Sam M, Peng-Jun W, Chih-Wei Y (2005). Power Assignment for k-Connectivity in Wireless Ad Hoc Networks. In 24th Annual Joint Conference of the IEEE Computer and Communications Societies (INFOCOM2005), Miami, Florida, USA.

Xu X, Liang W (2011). Placing optimal number of sinks in sensor networks for network lifetime maximization. In: Proc. IEEE ICC'11, 2011, June 5-9, pp. 1-6.

Yao-Chung C, Zhi-Sheng L, Jiann-Liang C (2006). Cluster Based SelfOrganization Management Protocols for Wireless Sensor Networks. IEEE Trans. Consum. Electron. 52(1):75-80. http://dx.doi.org/10.1109/TCE.2006.1605028 